\title{
Building an End User focused THz based Ultra High Bandwidth Wireless Access Network: The TERAPOD Approach
}

\author{
Alan Davy*, Luis Pessoa ${ }^{\dagger}$, Cyril Renaud ${ }^{\ddagger}$, Edward Wasige ${ }^{\S}$, Mira Naftaly ${ }^{\natural}$, Thomas Kürner",

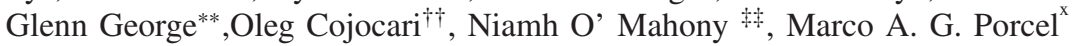 \\ *Telecommunications Software \& Systems Group, Waterford Institute of Technology, Ireland. adavy@tssg.org \\ ${ }^{\dagger}$ INESC TEC and Faculty of Engineering, University of Porto, Portugal. lpessoa@inesctec.pt \\ \$University College London, UK. c.renaud@ucl.ac.uk \\ $\S$ University of Glasgow, UK. Edward.Wasige@glasgow.ac.uk \\ IINational Physical Laboratory, Teddington, UK. mira.naftaly@npl.co.uk \\ "Technische Universitt Braunschweig, Germany. Kuerner@ifn.ing.tu-bs.de \\ **BBay Photonics, UK. glenn.george@ bayphotonics.com \\ ${ }^{\dagger}$ ACST GmbH, Germany. oleg.cojocari@acst.de \\ 中Dell EMC, Cork, Ireland. Niamh.Omahony@dell.com \\ ${ }^{x}$ VLC Photonics S.L., Spain. marco.garcia@vlcphotonics.com
}

\begin{abstract}
The TERAPOD project aims to investigate and demonstrate the feasibility of ultra high bandwidth wireless access networks operating in the Terahertz (THz) band. The proposed TERAPOD THz communication system will be developed, driven by end user usage scenario requirements and will be demonstrated within a first adopter operational setting of a Data Centre. In this article, we define the full communications stack approach that will be taken in TERAPOD, highlighting the specific challenges and aimed innovations that are targeted.

Index Terms-Terahertz Communication, Resonance Tunnelling Diodes, Uni-travelling Carrier PhotoDiode, Skotky Barrier Diode, Standardisation
\end{abstract}

\section{INTRODUCTION}

The TERAPOD project aims to investigate and demonstrate the feasibility of ultra high bandwidth wireless access networks operating in the Terahertz $(\mathrm{THz})$ band. The proposed TERAPOD THz communication system will be developed, driven by a selected set of end user usage scenario requirements. The project ambitiously aims to demonstrate the TERAPOD THz communication system within a first adopter operational setting of a Data Centre and will significantly progress innovations across the full $\mathrm{THz}$ communications system stack.

TERAPOD takes a multi-pronged approach to demonstrate the feasibility of $\mathrm{THz}$ communication within a Data Centre by leveraging breakthroughs recently made in $\mathrm{THz}$ devices, systems, metrology, protocols and standardization. The layered approach will ensure that all system requirements aligned to demonstration within a Data Centre will be integrated into development activities.

The remainder of this article is structured as follows; Section 2 highlights the current challenges and related work in the area of $\mathrm{THz}$ Communication. Section 3 outlines the TERAPOD approach to building an end to end THz communications system. Section 4 highlights the main innovations targeted by the TERAPOD project and expected impacts. Section 5 concludes the article by summarising the project targeted outcomes.

\section{RELATED WORK}

The demand for bandwidth in wireless communications has doubled every 18 months since 1980 following the wellknown Edholm's law [1]. A 2016 Cisco report [2] concludes that by 2020, Wi-Fi and mobile devices will account for 66 percent of all IP traffic up from 48 percent in 2015, while the global internet traffic is expected to grow three-fold between 2015 and 2020. This growing demand results essentially from increased traffic requirements from existing users, which translates into higher wireless data-rate requirements. In order to support these future demands, there is an urgent need to develop new technology platforms beyond currently available wireless technology where only marginal gains are possible.

With expected demands for $100 \mathrm{Gbit} / \mathrm{s}$ wireless connectivity in a near future, finding solutions in existing frequency bands below $100 \mathrm{GHz}$ is very challenging. Millimetre wave bands, around $60 \mathrm{GHz}$ and E-band $(71-95 \mathrm{GHz})$ are already being used for in-room data distribution and point-to-point backhaul of mobile communications data, and are being investigated for fixed-wireless access. For example, in the $60 \mathrm{GHz}$ band, 7 to 9 $\mathrm{GHz}$ of spectrum has been allocated worldwide. However, in order to achieve $100 \mathrm{Gbit} / \mathrm{s}$, spectral efficiencies of the order of $10-15 \mathrm{bit} / \mathrm{s} / \mathrm{Hz}$ have to be achieved, which is a huge technical challenge. 


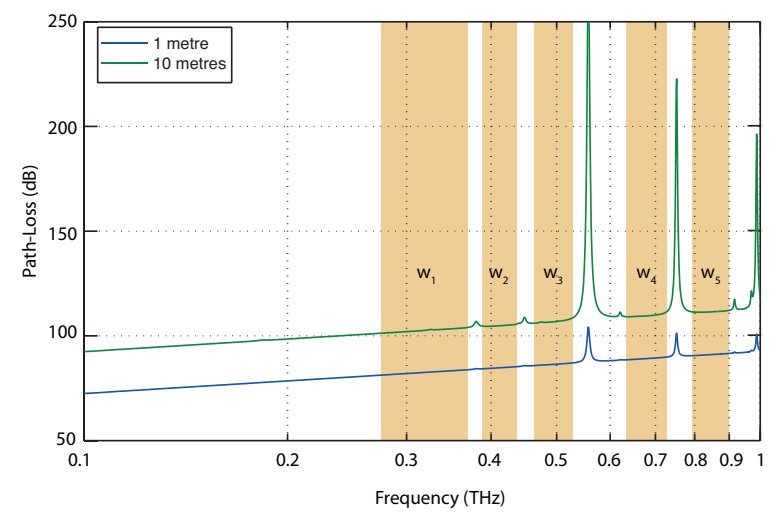

Fig. 1. Path-loss for wireless links of 1 and 10 meters between $100 \mathrm{GHz}$ and $1 \mathrm{THz}$

For a real step change towards the coveted $1 \mathrm{Tbit} / \mathrm{s}$ wireless transmission, new areas of the spectrum must be utilized. With the emergence of viable $\mathrm{THz}$ communications systems on the horizon, it is crucial to develop a technology roadmap for $\mathrm{THz}$ communication and networking for beyond the $5 \mathrm{G}$ timeframe. The huge bandwidths available in the terahertz spectrum allows for moderate spectral efficiencies to be employed for target data-rates of $100 \mathrm{Gbit} / \mathrm{s}$, which ultimately translates into reduced energy consumption of communication systems. Therefore, the exploitation of higher frequency spectrum regions to alleviate the spectrum scarcity and bandwidth limitations of current microwave systems is inevitable.

The terahertz band is still relatively unexplored for communication purposes, especially because the spectrum above 275 $\mathrm{GHz}$ is still unregulated, with no allocation to specific active services, although this is changing with emerging standardization efforts [3]. In the $\mathrm{THz}$ band below $1 \mathrm{THz}$, there are several transmission windows with low atmospheric losses. The main $\mathrm{THz}$ spectrum transmission window targeted in TERAPOD is around $300 \mathrm{GHz}$, which provides roughly $100 \mathrm{GHz}$ of bandwidth (W1), although several other windows up to $1 \mathrm{THz}$ will be considered as shown in pathloss.

Terahertz communications have the potential for important short-range applications, such as wireless local area networks (WLAN), high-speed wireless links between servers in a data centre [4], wireless high-definition TV transmissions, as well as for chip-to-chip communication, and machine-to-machine (M2M) communication (wireless personal area networks WPAN) [5].

The viability of $\mathrm{THz}$ wireless communications has been supported by recent developments and demonstrations using both electronic and photonic $\mathrm{THz}$ system components [6]. Currently the photonic-approach is considered as leading the development of wireless data links with very high data rates, thanks to the availability of telecom-based high-frequency components such as lasers, modulators and photomixers [7].

On the other hand, there are several semiconductor elec- tronic candidates for $\mathrm{THz}$ emitters or oscillators operating at room temperature such as tunnel transit-time (TUNNET) diodes, impact ionization avalanche transit-time (IMPATT) diodes, Gunn diodes, resonant tunnelling diodes (RTDs), and transistor-based oscillators [8]. Among the different competing technologies, RTDs have exhibited the highest oscillation frequencies at nearly $2 \mathrm{THz}$ [9]. Beyond RTDs, transistorbased monolithic microwave integrated circuits (MMICs) have also been demonstrated to be suitable for both transmitters and receivers, as well as schemes based on frequency multiplication [10]. In this regard, RTDs are very appealing since their simple circuitry allows for the change of operation between transmitter and receiver with a simple bias voltage adjustment. Regarding receivers, electronic approaches have been generally demonstrated, such as those based on Schottky diodes, as well as on RTDs [5].

\section{THE TERAPOD APPROACH}

TERAPOD pursues the ambitious vision of the short-range Tbit/s wireless connectivity paradigm, by exploiting three of the most promising emerging $\mathrm{THz}$ device technologies, namely (1) resonant tunnelling diodes, (2) uni-traveling-carrier photodiodes and (3) Schottky barrier diodes to enable the development and integration of the building blocks required for ultra-broadband communications in the $\mathrm{THz}$ spectrum. Therefore, TERAPOD employs an holistic approach where multiple technologies are explored simultaneously in order to identify the architectures where the advantages of each technology can be fully extracted instead of relying on each of the technologies separately. In fact not a single technology will be able to support the requirements of developing $\mathrm{THz}$ transceivers with high speed optical/wireless interfaces and electrical/interfaces, and receiver architectures.

Achieving the Tbit/s wireless connectivity paradigm requires the employment of very high frequency bands above $300 \mathrm{GHz}$ and up to $1 \mathrm{THz}$, since the frequency bands currently in use (below $100 \mathrm{GHz}$ ) do not seem sufficient to accommodate the predicted future data-rate requirements. In fact, while previous research below $100 \mathrm{GHz}$ has been focused on improving spectral efficiency as well as spatial efficiency (with MIMO and beamforming), the use of frequency bands where ultra-high bandwidth channels are available allows for relaxed spectral efficiency requirements which translates into reduced energy consumption, whereas the capacity scale-up of systems operating below $100 \mathrm{GHz}$ will certainly result in a linear scaleup in energy consumption which is problematic.

Putting this vision in place requires the employment of femtocell-based networks, possibly based on cloud radio access networks (Cloud-RAN) with centralized baseband processing, where short-range wireless connections allow for low power consumption transceivers having reduced transmission power and expectedly very high energy efficiency. Depending on the use case, the requirement for a densely deployed short-range wireless network capable of providing adequate wireless coverage, may dictate the need of finding costeffective transceiver solutions, so that the data-rates can be 
increased in line with future predicted requirements without a linear cost increase from communication infrastructures. Furthermore, supporting such concepts with simple and integrated interfaces between fibre and wireless is essential in a cost and maintenance perspective.

To achieve the proposed target, TERAPOD proposes to exploit the merits of 3 different technologies, namely RTDs, UTC-PDs and SBDs, in order to develop the building blocks (or artefacts) required for ultra-high bandwidth $\mathrm{THz}$ based communications, such as, sources/oscillators, mixers and detectors:

- RTDs are devices implemented on MMIC technology. RTDs can serve both source and detector functions and are currently the fastest purely electronic devices with oscillation frequencies capable of reaching nearly $2 \mathrm{THz}$, at room temperature. Unlike traditional approaches, the RTD intrinsic gain allows for a simple and energy efficient implementation, avoiding the use of complex transceiver building blocks.

- Uni-Travelling Carrier Photo-Diodes (UTC-PDs) are photonic based sources that have provided world record, room temperature, $\mathrm{CW}$ output powers up to $\mathrm{THz}$ frequencies, and given their photonic nature, can provide ultrawide band frequency tuneability and natural convergence with optical network.

- Schottky barrier diodes are a technology of choice for compact and low-cost receivers up to the THz range. They can be used as envelope detectors for simple modulation formats, but can also be used as mixers in heterodyne receivers with the advantage of a much higher sensitivity and possibility of phase modulation.

TERAPOD leverages the recent developments in each of these technologies in order to develop the required ultra-high bandwidth $\mathrm{THz}$ communications building blocks and push the state of the art in this area by addressing the development of substrate integrated $\mathrm{THz}$ antennas, device arraying fed by a photonics integrated circuit phase distribution for increased output power and beam forming.

\section{TARGETED INNOVATIONS}

TERAPOD is focused on the development of several technologies, components, communications methods and architectures and protocols aiming to answer an emerging necessity of the market in the near future. In this sense, the project targets a proof-of-concept in a Datacenter deployment, that will be ultimately achieved using a small scale test-bed for the demonstration of at least 100 Gbps wireless communications between several stand-alone prototype nodes to be developed and manufactured within the project, including both optoelectronic and all-electronic interconnects at $\mathrm{THz}$ frequencies, in an operational environment.

Figure terapodScheme provides a graphical description of the overall approach. The TERAPOD approach relies on developing the necessary components, devices, metrology and protocols to enable the deployment of the proposed technology in $100 \mathrm{Gbps} \mathrm{THz}$ short-range wireless communications in short term and paving the way for $1 \mathrm{Tbit} / \mathrm{s}$ in the long term. TERAPOD significantly innovates upon the state of the art in ultra-broadband communications using $\mathrm{THz}$ frequency bands. Specific contributions are detailed below:

A. Demonstration of reliable, high efficiency and high-power THz RTD sources

THz operation of RTD oscillators has been demonstrated by a few laboratories, with fundamental oscillations up to $1.92 \mathrm{THz}$ recently being demonstrated [9], [11]. The main limitation of RTD oscillators has been their low output power (in the tens of microwatts range), but relatively high output power for some of these oscillators is now being reported, e.g. up to $1 \mathrm{~mW}$ at $300 \mathrm{GHz}$ with large micron-sized devices (by members of the present consortium) [12] and $0.6 \mathrm{~mW}$ at 620 $\mathrm{GHz}$ with a two-element array by a Japanese group [13]. RTDs with $1 \mathrm{~mW}$ at $300 \mathrm{GHz}$ were developed on the H2020 iBROW project including oscillators with record output powers in the $0.5-1 \mathrm{~mW}$ range at $300 \mathrm{GHz}$ [12], [14], [15]. Suitable antennas for integration with these oscillators have also been developed [16].

TERAPOD will innovate upon the state-of-the-art of RTD technology by building on top of the results of iBROW in several aspects: - Address the manufacturability and reliability of the baseline $300 \mathrm{GHz}$ RTD device and oscillator technology. - Deliver a highly efficient $\mathrm{THz}$ technology. - Demonstrate RTD operation as voltage controlled oscillator (VCO) by employing varactor diodes as recently first demonstrated by a Japanese group - Demonstrate a novel RTD epitaxial layer structure that provides improved efficiency and output power by maximing the negative differential conductance (NDC) region.

B. Demonstration of low-barrier diodes for operation as $\mathrm{THz}$ mixer

While modern MMIC technology does not provide highlyintegrated receiver solutions at SubMM-Waves, SchottkyDiode-based Envelope Detector is often the technology of choice for compact and low-cost receivers. Paving the way for the Tbit/s wireless connectivity will certainly require not only the operation in $\mathrm{THz}$ frequency bands but also more spectrally efficient modulation formats. For such links, one would need a coherent heterodyne receiver. Normally such a receiver requires relatively high Local-Oscillator (LO) power. Recent developments suggest feasibility of frequency mixers based on low-barrier diodes, which require just a fraction of the LO-power as required by traditional mixers [17].

TERAPOD will innovate upon the state-of-the-art by building over preliminary results that suggest acceptable performance of a $183 \mathrm{GHz}$ mixer while using just $200 \mathrm{tW}$ of LOpower in order demonstrate a low-barrier diode suitable for operation as a $\mathrm{THz}$ mixer.

C. Demonstration of power combination of multiple $\mathrm{THz}$ sources

Although recent demonstrations have proven the capacity of $\mathrm{THz}$ communication system for high throughput, most of 


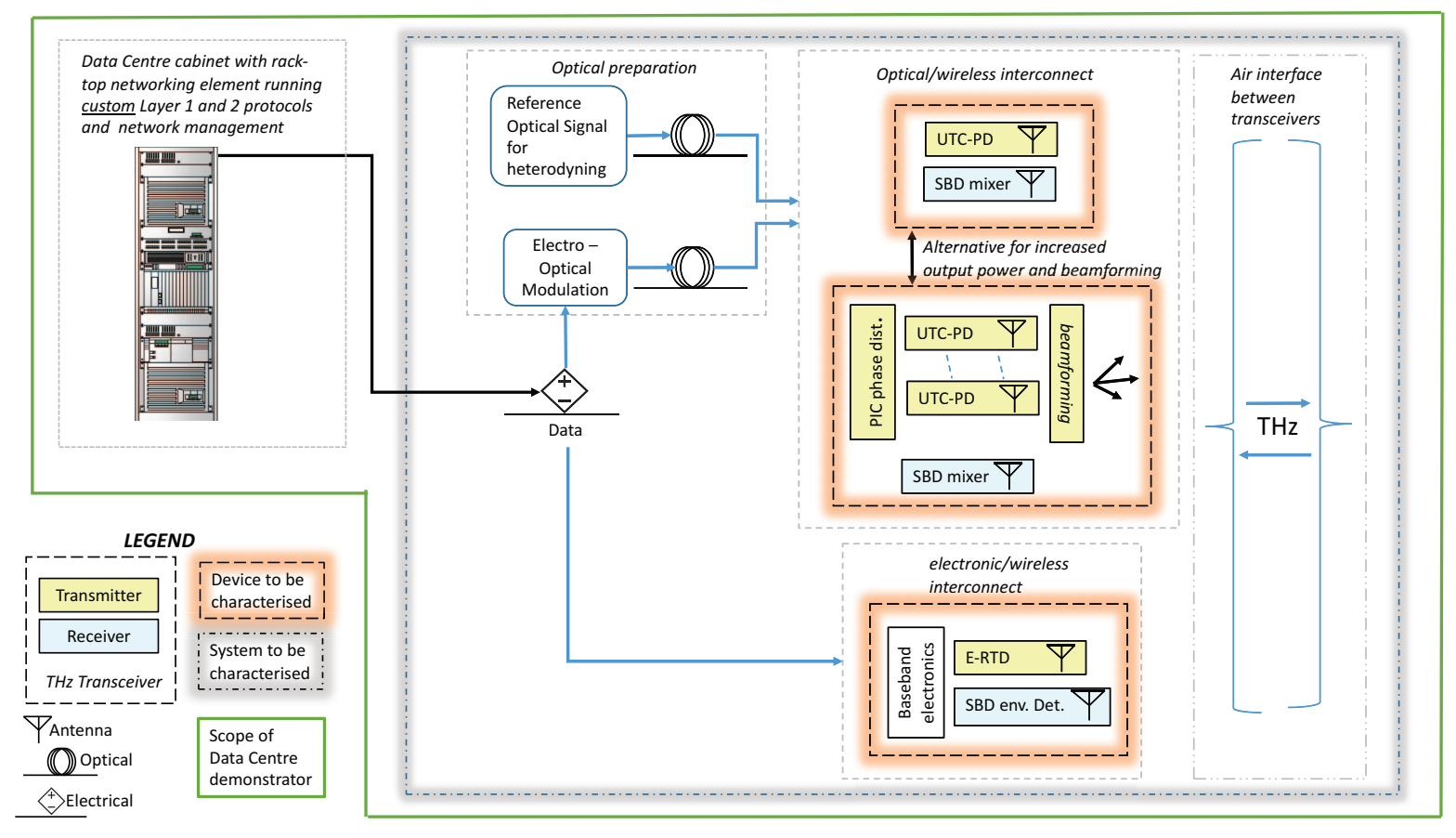

Fig. 2. Schematic representation of the TERAPOD technology application scenario

these system have not been tested within a real application environment and are still limited by the performances of the different components. In fact, the intrinsic high propagation loss at increasing carrier frequencies and the low power generated at these frequencies limits the $\mathrm{THz}$ wireless transmission distances to typically a few metres.

As $\mathrm{THz}$ source devices require a small area to achieve high speed performance, this limits the achievable saturation power, which demonstrates that such devices are hard to create. So far, the best output power achieved is around $1 \mathrm{~mW}$ at $300 \mathrm{GHz}$ for both UTC-PDs [18] and RTDs [14]. To further increase the output power, two or more devices could be combined and this has been done with two UTC-PDs providing more than $1 \mathrm{~mW}$ at $300 \mathrm{GHz}$ [19], but quite close to device destruction.

TERAPOD will innovate upon the state-of-the-art by demonstrating that the output of several UTC-PDs can be combined ultimately using an approach based on antenna arrays integrated with a photonic phase distribution systems, which could also allow beam-steering offering the possibility for electrical steering of receiver antenna and automatic search of the transmitter antenna. This has yet to be demonstrated at $\mathrm{THz}$ frequencies, particularly beyond $200 \mathrm{GHz}$ but has been reported for a four-element linear array at $88 \mathrm{GHz}$ [20].

\section{Demonstration of novel measurement and characterisation} techniques for $\mathrm{THz}$ devices

As yet, there is no standardised traceable metrology for $\mathrm{THz}$ devices. THz transmitter power radiated into free space is commonly measured using power meters such as pyroelectric,
Golay cell, or thermopile [21], [22]. The output frequency of UTCs is seldom measured directly, but is instead calculated as the difference-frequency of the two source lasers [23]. The frequency of electronic sources such as RTDs, must be measured directly, i.e. by a spectrometer device [13]. Depending on the type, these may have a limited frequency resolution and/or bandwidth, and sometimes frequency-dependent response. Measurements of transmitter and receiver patterns have long been employed for RF antennas [24] but have never been implemented for $\mathrm{THz}$ devices. $\mathrm{THz}$ beam propagation has been measured in outdoor and indoor environments [25]; these, however, were generic environments.

TERAPOD will innovate upon the state-of-the-art by developing metrology techniques and instrumentation for characterisation of $\mathrm{THz}$ devices. The absolute emitted power will be measured using a traceably calibrated power meter recently developed and tested by PTB [26]. The beam divergence will also be measured, and the power density as a function of distance from the emitter will be calculated. A customdesigned lamellar interferometer [27] with broadband response (0.1-10 THz) and high frequency resolution $0.3 \mathrm{GHz}$ will be used in conjunctions with a calibrated power meter to determine the power spectral density of the emitted radiation, with particular attention to the possible presence of sidebands or higher harmonics and their effect on the fundamental signal. Spectral responsivity of $\mathrm{THz}$ receivers will likewise be measured using the interferometer and a power-calibrated broadband source. The radiation pattern of $\mathrm{THz}$ transmitters and the acceptance pattern of $\mathrm{THz}$ receivers will also be 
determined. A first $\mathrm{THz}$ transmission test chamber will be constructed with ambient conditions similar to those in the target deployment scenarios.

\section{E. Demonstration of novel substrate integrated $\mathrm{THz}$ antennas}

$\mathrm{THz}$ antenna solutions are typically based on the employment of silicon lenses [28], [29]. The high permittivity of the substrate leads to propagating modes and reflections within the substrate. It implies an unpredictable radiation pattern as a function of frequency. The employment of silicon lenses improves the beam shape and radiation pattern while providing broadband focusing properties. However, when beamforming characteristics are desired, lens-based solutions require mechanical lens adjustment which is complex and prone to failure. In order to solve these problems, Brown and Parker [30] investigated for the first time the use of photonic crystals as a substrate for planar antennas. TERAPOD will innovate upon the state-of-the-art by exploring photonic bandgap (PBG) crystal structures to achieve precise control over the propagation of the electromagnetic waves in the $\mathrm{THz}$ region, which when combined with an optical phase distribution chip will allow for beamforming, therefore avoiding mechanical based adjustment solutions for achieving control over the beam. Their employment and integration in RTD, UTC-PD and SBD based devices will be addressed in TERAPOD for the first time.

\section{F. Demonstration of novel higher layer $\mathrm{THz}$ communications protocols}

Due to the need for high-gain antennas on both ends of the $\mathrm{THz}$ wireless link, device discovery and beam searching are putting new challenges to higher layer protocols. One possibility to accelerate the corresponding process is to use a second communication channel at a lower frequency allowing antennas with larger beamwidths to have a rough estimation of the positions of TX and RX.ă First simulation-based studies of such concepts applied to simple environments have been published in [31]. Due to the impact of atmospheric absorption across the $\mathrm{THz}$ spectrum, optimal frequency selection strategies in a frequency division medium access regime can reduce path loss and improve throughput [32].

TERAPOD will innovate upon state of the art in physical layer, data link layer and network layer protocols by developing a series of simulator models of the $\mathrm{THz}$ communication system stack accounting for TERAPOD device performance characteristics and operational environment channel measurements. Physical layer protocols for device discovery and beam steering will be evaluated; medium access protocols (frequency division, time division) along with coherent coding techniques will be evaluated from an information theoretic stand point; flexible network resource management strategies based on Software Defined Networking will be evaluated to take advantage of $\mathrm{THz}$ communication links.

\section{CONCLUSION}

The TERAPOD Project is an ambitious 3 year project which sets its objectives on demonstrating an operational ready $\mathrm{THz}$ communication system within a Data Centre environment. In doing so, TERAPOD aims to progress the technology readiness level of $\mathrm{THz}$ communications systems closer to industry adoption; advance non technical barriers to the adoption of $\mathrm{THz}$ communication technology and accelerate the growth of $\mathrm{THz}$ communications research and development both in Europe and Internationally. TERAPOD seeks to make a number of crucial technical innovations to see its objectives achieved. These are in areas such as $\mathrm{THz}$ devices and components, $\mathrm{THz}$ metrology and characterisation, $\mathrm{THz}$ communications protocols and finally standardisation and regulation.

\section{ACKNOWLEDGMENT}

This project has received funding from the European Union's Horizon 2020 research and innovation programme under grant agreement 761579 (TERAPOD).

\section{REFERENCES}

[1] S. Cherry, "Edholm's law of bandwidth," IEEE Spectrum, vol. 41, no. 7, pp. 58-60, 2004

[2] Cisco. Visual networking index. [Online]. Available: http://www.cisco.com/c/en/us/solutions/collateral/serviceprovider/visual-networking-index-vni/vni-hyperconnectivity-wp.html

[3] T. Kürner and S. Priebe, "Towards thz communications-status in research, standardization and regulation," Journal of Infrared, Millimeter, and Terahertz Waves, vol. 35, no. 1, pp. 53-62, 2014.

[4] B. Peng and T. Kürner, "A stochastic channel model for future wireless thz data centers," in Wireless Communication Systems (ISWCS), 2015 International Symposium on. IEEE, 2015, pp. 741-745.

[5] K. Ishigaki, M. Shiraishi, S. Suzuki, M. Asada, N. Nishiyama, and S. Arai, "Direct intensity modulation and wireless data transmission characteristics of terahertz-oscillating resonant tunnelling diodes," Electronics letters, vol. 48, no. 10, pp. 582-583, 2012.

[6] T. Nagatsuma, G. Ducournau, and C. C. Renaud, "Advances in terahertz communications accelerated by photonics," Nature Photonics, vol. 10, no. 6, pp. 371-379, 2016.

[7] T. Nagatsuma, S. Horiguchi, Y. Minamikata, Y. Yoshimizu, S. Hisatake, S. Kuwano, N. Yoshimoto, J. Terada, and H. Takahashi, "Terahertz wireless communications based on photonics technologies," Optics express, vol. 21 , no. 20 , pp. 23736-23747, 2013.

[8] T. Nagatsuma, "Terahertz communications technologies based on photonic and electronic approaches," in Wireless Conference (European Wireless), 2012 18th European. VDE, 2012, pp. 1-4.

[9] T. Maekawa, H. Kanaya, S. Suzuki, and M. Asada, "Oscillation up to 1.92 thz in resonant tunneling diode by reduced conduction loss," Applied Physics Express, vol. 9, no. 2, p. 024101, 2016.

[10] J. Federici and L. Moeller, "Review of terahertz and subterahertz wireless communications," Journal of Applied Physics, vol. 107, no. 11, p. $6,2010$.

[11] J. Lee, M. Kim, and K. Yang, "A 1.52 thz rtd triple-push oscillator with a w-level output power," IEEE Transactions on Terahertz Science and Technology, vol. 6, no. 2, pp. 336-340, 2016.

[12] J. Wang, A. Al-Khalidi, K. Alharbi, A. Ofiare, H. Zhou, E. Wasige, and J. Figueiredo, "High performance resonant tunneling diode oscillators as terahertz sources," in Microwave Conference (EuMC), 2016 46th European. IEEE, 2016, pp. 341-344.

[13] S. Suzuki, M. Shiraishi, H. Shibayama, and M. Asada, "High-power operation of terahertz oscillators with resonant tunneling diodes using impedance-matched antennas and array configuration," IEEE Journal of Selected Topics in Quantum Electronics, vol. 19, no. 1, pp. 8500108 $8500108,2013$.

[14] K. H. Alharbi, "High performance terahertz resonant tunnelling diode sources and broadband antenna for air-side radiation," Ph.D. dissertation, University of Glasgow, 2016.

[15] E. Wasige, A. Al-Khalidi, K. Alharbi, and J. Wang, "High performance microstrip resonant tunneling diode oscillators as terahertz sources," in Millimetre Waves and Terahertz Technologies (UCMMT), 2016 IEEE 9th UK-Europe-China Workshop on. IEEE, 2016, pp. 25-28. 
[16] K. H. Alharbi, A. Khalid, A. Ofiare, J. Wang, and E. Wasige, "Diced and grounded broadband bow-tie antenna with tuning stub for resonant tunnelling diode terahertz oscillators," IET Microwaves, Antennas \&amp; Propagation, vol. 11, no. 3, pp. 310-316, 2016.

[17] S. Khanal, T. Kiuru, M. Hoefle, J. Montero, O. Cojocari, J. Mallat, P. Piironen, and A. V. Räisänen, "Characterisation of low-barrier schottky diodes for millimeter wave mixer applications," in Millimeter Waves (GSMM) \&amp; ESA Workshop on Millimetre-Wave Technology and Applications, 2016 Global Symposium on. IEEE, 2016, pp. 1-4.

[18] A. Wakatsuki, T. Furuta, Y. Muramoto, T. Yoshimatsu, and H. Ito, "High-power and broadband sub-terahertz wave generation using a jband photomixer module with rectangular-waveguide output port," in Infrared, Millimeter and Terahertz Waves, 2008. IRMMW-THz 2008. 33 rd International Conference on. IEEE, 2008, pp. 1-2.

[19] H.-J. Song, K. Ajito, Y. Muramoto, A. Wakatsuki, T. Nagatsuma, and N. Kukutsu, "Uni-travelling-carrier photodiode module generating $300 \mathrm{ghz}$ power greater than $1 \mathrm{mw}, "$ IEEE Microwave and Wireless Components Letters, vol. 22, no. 7, pp. 363-365, 2012.

[20] T. P. McKenna, J. A. Nanzer, and T. R. Clark, "Photonic beamsteering of a millimeter-wave array with $10 \mathrm{gb} / \mathrm{s}$ data transmission," IEEE Photon. Technol. Lett., vol. 26, no. 14, pp. 1407-1410, 2014.

[21] H. Ito, T. Yoshimatsu, H. Yamamoto, and T. Ishibashi, "Widely frequency tunable terahertz-wave emitter integrating uni-traveling-carrier photodiode and extended bowtie antenna," Applied Physics Express, vol. 6, no. 6, p. 064101, 2013.

[22] X. Yu, R. Asif, M. Piels, D. Zibar, M. Galili, T. Morioka, P. U. Jepsen, and L. K. Oxenløwe, "400-ghz wireless transmission of 60 -gb/s nyquistqpsk signals using utc-pd and heterodyne mixer," IEEE Transactions on Terahertz Science and Technology, vol. 6, no. 6, pp. 765-770, 2016.

[23] J.-M. Wun, H.-Y. Liu, Y.-L. Zeng, S.-D. Yang, C.-L. Pan, C.-B. Huang, and J.-W. Shi, "Photonic high-power continuous wave thz-wave generation by using flip-chip packaged uni-traveling carrier photodiodes and a femtosecond optical pulse generator," Journal of Lightwave Technology, vol. 34 , no. 4, pp. 1387-1397, 2016

[24] C. Li, T.-H. Loh, Z. H. Tian, Q. Xu, and Y. Huang, "A comparison of antenna efficiency measurements performed in two reverberation chambers using non-reference antenna methods," in Antennas \&amp; Propagation Conference (LAPC), 2015 Loughborough. IEEE, 2015, pp. 1-5.

[25] S. Priebe, C. Jastrow, M. Jacob, T. Kleine-Ostmann, T. Schrader, and T. Kurner, "Channel and propagation measurements at 300 ghz," IEEE Transactions on Antennas and Propagation, vol. 59, no. 5, pp. 16881698, 2011.

[26] A. Steiger, R. Müller, A. R. Oliva, Y. Deng, Q. Sun, M. White, and J. Lehman, "Terahertz laser power measurement comparison," IEEE Transactions on Terahertz Science and Technology, vol. 6, no. 5, pp. 664-669, 2016.

[27] M. Naftaly, P. Dean, R. E. Miles, J. R. Fletcher, and A. Malcoci, "A simple interferometer for the analysis of terahertz sources and detectors," IEEE journal of selected topics in quantum electronics, vol. 14 , no. 2 , pp. 443-448, 2008.

[28] A. J. Seeds, M. J. Fice, K. Balakier, M. Natrella, O. Mitrofanov, M. Lamponi, M. Chtioui, F. van Dijk, M. Pepper, G. Aeppli et al., "Coherent terahertz photonics," Optics express, vol. 21, no. 19, pp. 22 988-23 000, 2013.

[29] M. Asada and S. Suzuki, "Compact thz oscillators with resonant tunneling diodes and application to high-capacity wireless communications," in Applied Electromagnetics and Communications (ICECom), 201321 st in Applied Electromagnetics and Communications (ICEC
International Conference on. IEEE, 2013, pp. 1-5.

[30] E. Brown, C. Parker, and E. Yablonovitch, "Radiation properties of a planar antenna on a photonic-crystal substrate," JOSA B, vol. 10, no. 2 , pp. 404-407, 1993.

[31] B. Peng, S. Priebe, and T. Kürner, "Fast beam searching concept for indoor terahertz communications," in Antennas and Propagation (EuCAP), 2014 8th European Conference on. IEEE, 2014, pp. 639-643.

[32] A. Afsharinejad, A. Davy, and B. Jennings, "Dynamic channel allocation in electromagnetic nanonetworks for high resolution monitoring of plants," Nano Communication Networks, vol. 7, pp. 2-16, 2016. 